\title{
Localization Length Exponent, Critical Conductance Distribution and Multifractality in Hierarchical Network Models for the Quantum Hall Effect
}

\author{
Andreas Weymer, Martin Janssen \\ Institut für Theoretische Physik, Universität zu Köln, \\ Zülpicher Strasse 77, 50937 Köln, Germany
}

(May 05, 1998)

\begin{abstract}
We study hierarchical network models which have recently been introduced to approximate the Chalker-Coddington model for the integer quantum Hall effect (A.G. Galstyan and M.E. Raikh, PRB 561422 (1997); Arovas et al., PRB 56, 4751 (1997)). The hierarchical structure is due to a recursive method starting from a finite elementary cell. The localization-delocalization transition occurring in these models is displayed in the flow of the conductance distribution under increasing system size. We numerically determine this flow, calculate the critical conductance distribution, the critical exponent of the localization length, and the multifractal exponents of critical eigenstates.
\end{abstract}

PACS numbers: 73.23.-b Mesoscopic systems; 73.40.Hm Quantum Hall effect (integer and fractional); 64.60.Ak Renormalization-group, fractal, and percolation studies of phase transitions

\section{INTRODUCTION}

Localization brought about by quantum interference in disordered media [1] is an ongoing research topic. One reason for this lies in the complex structure of the associated localization-delocalization (LD) transition, a quantum phase transition which has no simple mean field description [2]. Although many aspects of the LD transition are understood and quantitative results for critical exponents have been obtained by extensive numerical studies (see e.g. [3 ) there is no complete theory available yet. Best understood are quasi-one-dimensional (1D) systems, where the notion of a transfer-matrix led to strong analytic methods (see e.g. [4,5]). Non-perturbative renormalization techniques have also been developed on the basis of quasi-1D geometries. However, no such technique proved to be successful directly for higher dimensional systems. There have been early attempts in that direction [6],7] based on real-space renormalization within a Hamiltonian description of the systems dynamics. A major obstacle against their success was the increase in the number of relevant matrix elements of the effective Hamiltonian after a few renormalization-group (RG) steps - unless the system was in a regime of strong localization. Only recently two groups have independently introduced a novel real-space renormalization technique for the LD problem [8,9]. It was realized that a local scattering theoretical formulation (see e.g. [10]) of the systems dynamics gives rise to a straightforward real-space $\mathrm{RG}$ which is not restricted to quasi-1D. In particular, they considered the Chalker-Coddington (CC) model [11], a two-dimensional network of nodes and links where waves can propagate on the links and are scattered by chiral scatterers placed on the nodes. This model is very convenient for testing the RG ideas since the scatterers are described by simple $2 \times 2$ random $S$-matrices. It has been established 11 13 that the $\mathrm{CC}$ model contains interesting LD physics. The chiral structure is meant to capture the essential features of the motion of $2 \mathrm{D}$ electrons in a strong perpendicular magnetic field and a random potential 11]. Such electron systems exhibit the integer quantum Hall effect and are denoted as quantum Hall systems [14. The quantum Hall effect is essentially caused by a LD transition [15]. In a localized state the electron is confined to some finite area within the bulk of the system while the electron's wave function is extended over the entire system in a delocalized state. However, states carrying a unidirectional quantized edge-current exist also in the localized phase of a quantum Hall system. The link amplitudes of the CC model can be identified with local wave functions of a quantum Hall system. On increasing the average scattering strength $\langle T\rangle$ from 0 to 1 one finds localization and chiral edge states (carrying a quantized Hall current) in almost any case. However, as soon as $\langle T\rangle$ is close to 0.5 extended bulk states appear, and e.g. the Hall conductance jumps to another quantized plateau value. It is expected that, in the thermodynamic limit, the transition occurs at precisely $\langle T\rangle=T^{*}=0.5$. At the plateau to plateau transition the localization length $\xi$ of a critical state diverges, $\xi \propto\left|\langle T\rangle-T^{*}\right|^{-\nu}$, where the critical exponent $\nu=2.35 \pm 0.03$ [16]. Furthermore, it is known that the states are multifractal as soon as their localization length becomes larger than system size [13.

It turned out, that the qualitative aspects of the LD transition could be described by a simple MigdalKadanoff type approach [9] leading to simple algebraic RG equations. Critical exponents, however, were found to be different from the commonly accepted values. Quantitatively more reliable results were obtained by \& when using a slightly more realistic approximation to the CC model. In that case, however, the treatment needed some numerical assistance. Despite the success in describing the LD transition the RG approach was based on uncontrolled approximations.

It was therefore tempting to elaborate further on the RG ideas of 8,9] and develop a general scheme which en- 
ables to improve quantitative results in a systematic manner. Advantageous for that purpose is the interpretation of the RG in terms of hierarchical networks embedded in the CC model. The hierarchical models are constructed from elementary cells cut out of the $\mathrm{CC}$ model and are characterized by the number of nodes, $b^{2}$, of the elementary cell and a number of active scatterers, $V$. For each model the flow of the conductance distribution, the critical conductance distribution and the critical exponent of the localization length can be determined by iterative RG steps. Furthermore, a calculation of multifractal properties of critical states in the hierarchical models allows to complete the description of LD transition characteristics. It turns out that our RG approach leads to reasonable values already for moderate sizes of elementary cells.

We have organized the paper as follows. In Sec. 2 we introduce the hierarchical models and describe their connection to those considered in [8,9]. In Sec. 3 the flow of the conductance distribution is analyzed. The critical exponent of the localization length and the critical conductance distributions are determined. The multifractal analysis of critical states in hierarchical models forms the content of Sec. 4, followed by our conclusions in Sec. 5 .

\section{HIERARCHICAL NETWORK MODELS}

We recall the construction of the Chalker-Coddington (CC) network model. A set of elementary scatterers is put on the nodes of a regular 2D lattice. Unidirectional channels link the scatterers to each other as shown in Fig. 1. The elementary scatterers are described by a unitary $2 \times 2$ scattering matrix $S$,

$$
\left(\begin{array}{c}
O \\
O^{\prime}
\end{array}\right)=S\left(\begin{array}{c}
I \\
I^{\prime}
\end{array}\right), \quad S=\left(\begin{array}{cc}
t & r^{\prime} \\
r & t^{\prime}
\end{array}\right)
$$

$T=|t|^{2}=\left|t^{\prime}\right|^{2}$ is the transmission to the left and $R=|r|^{2}=\left|r^{\prime}\right|^{2}=1-T$ is the transmission to the right. The phases on the links are taken as random variables uniformly distributed between 0 and $2 \pi$. Also the transmission ('scattering strength') $T \in[0,1]$ can be taken from a certain distribution.

To study the localization-delocalization behavior of the CC network model one can impose boundary conditions for a square network of linear size $L$ such that only total transmission to the left $T(L)$ or right $R(L)=1-T(L)$ is possible. In an appropriate two-probe conductance measurement (see Fig. 2) the transmission $T(L)$ yields the conductance $G$ via the Landauer-Büttiker theory of coherent transport,

$$
G=\frac{e^{2}}{h} T
$$

Thus, an interesting object to study the LD transition is the distribution function $P_{L}(T)$ and its flow with increasing $L$. In the thermodynamic limit, $L \rightarrow \infty$, bulk localized phases are characterized by $T=0$ or $T=1$. In both cases current can only flow along edge states. Thus, two stable (attractive) fixed point distributions $P_{\mathrm{I}}(T)=\delta(T)$ and $P_{\mathrm{II}}(T)=\delta(T-1)$ exist. The LD transition will be characterized by a third fixed point distribution, denoted as $P^{*}(T)$, which is unstable (repulsive). The unstable character means that $P^{*}$ is the limiting distribution, $\lim _{L \rightarrow \infty} P_{L}(T)=P^{*}(T)$, only if the set of its relevant parameters $X_{i}(L)$ (e.g. average value, median, variance) is initially taken to the fixed point values $X_{i}^{*}$. In any other case the distribution approaches one of the two stable fixed point distributions, $P_{\mathrm{I}, \mathrm{II}}$. For large enough initial sizes $L_{0}$ it may turn out that one parameter $X\left(L_{0}\right)$ is enough to fix the flow of the whole distribution. This situation is referred to as oneparameter-scaling regime. The corresponding $\beta$-function $\beta(X)=d X / d \ln L$ describes the scaling flow of $X(L)$ and allows for calculating the critical exponent $\nu$ of the correlation length (localization length) [17,

$$
\xi \propto\left|X-X^{*}\right|^{-\nu},
$$

from

$$
\nu^{-1}=\left.\frac{d \beta}{d X}\right|_{X^{*}}
$$

Therefore, a possible way of determining the critical exponent $\nu$ would be to set up a renormalization-group (RG) process for the flow of $P_{L}(T)$. An exact process could be achieved by recording the values $T(L)$ for arbitrary system sizes $L$ and for an arbitrary large number of realizations of the random scatterers. Such process could be carried out with the help of numerical calculations and is only limited by the computational capacity. In a more efficient way, however, one replaces the network model by some hierarchically constructed network that allows to perform the renormalization recursively. The hierarchical structure requires some finite elementary cell which allows for calculating the corresponding transmission $T$ analytically or by simple numeric routines. However, the replacement of the original network by a hierarchical one introduces some error which is not under control. To reach definite answers for critical exponents one has to repeat the RG process for larger and larger elementary cells such that eventually the original model can be recovered. This procedure is advantageous if (i) one is aiming at qualitative results and (ii) the results for the critical exponents rapidly converge. To get qualitative results about the LD transition the elementary cell should be as simple as possible, still containing essentials like basic symmetries. For example, the chiral structure and paths with loops in the CC network model should be maintained in order to allow for quantum interference along oriented paths. This idea was used in [9] where a simple elementary cell with 4 active scatterers (those for which neither $T=0$ nor $T=1$ ) was considered (see Fig. 6 in [9]). In that approximation the scattering turned out 
to obey one-dimensional composition laws which helped to proceed analytically. Unfortunately, in this approximation a discrete mirror symmetry (with respect to the central vertical axis) is broken. As a result, the critical distribution $P^{*}(T)$ is not symmetric with respect to $T=0.5$, and the critical average transmission is different from 0.5 .

The obvious choice for an elementary cell respecting also the mirror symmetry is shown in Fig. 3a. It consists of a $3 \times 3$ lattice containing 5 active scatterers and 4 scatterers (in the corners) that fully transmit to the left $(T=1)$ or to the right $(T=0)$. Building a hierarchical network from such elementary cells leads to a fractal network with fractal dimension $D^{[3 / 5]}=\ln 5 / \ln 3 \approx 1.46$ (see Fig. 3a). It will be denoted as [3/5]-model. The corresponding $\mathrm{RG}$ process has been already carried out in Ref. [8]. In that work a different interpretation was adopted. As in the [3/5]-model an elementary cell of 5 scattering units is replaced by one in each renormalization step(see Fig. 2 in [8]). As shown in Fig. $3 b$ the scaling factor is 2 instead of 3 . In other words, the RG process of [8] proceeds by constructing a new network from renormalized scatterers while our process considers a hierarchical network as a fractal subset of the original CC network. Apart from the different interpretation both processes are, of course, equivalent if the difference in scaling factors is taken into account. The simplified elementary cell, considered in Fig. 6 of [9] corresponds to the $[3 / 5]$-network when replacing the central scatterer by a fully reflecting/transmitting one (see Fig.3 c) and will henceforth be denoted as [3/4]-model. Its fractal dimension is $D^{[3 / 4]}=\ln 4 / \ln 3 \approx 1.26$.

In the present work we extend the RG process to larger elementary cells and study also the multifractal properties of critical wave functions.

A generalization to larger elementary cells is straightforward: each Chalker-Coddington network with $b \times b$ sites $(b=5,7,9 \ldots)$ and boundary conditions of only 4 links to the outer world can serve as an elementary cell. By recursion one gets a hierarchical infinite network (see Fig. 3a,c). Its fractal dimension is

$$
D^{[b / V]}=\frac{\ln V}{\ln b}
$$

where the elementary cell contains $V=\left(b^{2}+1\right) / 2$ scatterers. Note, that for $b \rightarrow \infty$ the original CC model is recovered and e.g. the fractal dimension approaches 2 for $b \gg 1$.

In general, hierarchical networks can be characterized by an odd number $b$ describing the size of its elementary cell $\left(b^{2}\right)$ and the number $V$ of active scatterers. Accordingly we denote the hierarchical networks as $[b / V]$ network.

In addition to the hierarchical networks with $V(b)=$ $\left(b^{2}+1\right) / 2$ which, for large $b$, reduce to the original $\mathrm{CC}$ model one can use $[b / V]$-elementary cells to construct larger elementary cells of size $b^{\prime} \times b^{\prime}$ with $V\left(b^{\prime}\right)<$ $\left(b^{\prime 2}+1\right) / 2$ active scatterers, in order to simplify the RG steps. We elaborated on a [9/37]-model constructed from [3/5]-elementary cells (displayed in Fig. 8). Note that the number of active scatterers $(V(9)=37)$ is less then $\left(9^{2}+1\right) / 2=41$ which would follow from the straightforward construction for $b=9$.

\section{FLOW OF THE CONDUCTANCE DISTRIBUTION}

After having outlined which hierarchical models will be considered in this work we are now going to describe how the RG for the conductance distribution was obtained in our numerical calculations.

1. In the first step we algebraically solve the scattering problem for a particular elementary cell. We obtain the transmission probability $T(L=b)$ as a function of the scattering matrix elements $t=|t| e^{i \alpha}, t^{\prime}=|t| e^{i \alpha^{\prime}}, r=$ $|r| e^{i \beta}$ and $r^{\prime}=|r| e^{i \beta^{\prime}}$ of all active scatterers. Here the unitarity constraints

$$
e^{i\left(\alpha+\alpha^{\prime}\right)}=-e^{i\left(\beta+\beta^{\prime}\right)}
$$

and

$$
|t|^{2}+|r|^{2}=1
$$

have to be obeyed. That leaves for $T(b)$ an algebraic expression of $V$ scattering strengths, $T_{1, \ldots, V}$, and $3 V$ phases.

2. In the second step we draw the scattering strengths $T$ from an initial distribution, $P_{0}(T)$ peaked around a certain mean value $\langle T\rangle_{0}$ and random uncorrelated phases for $\alpha, \alpha^{\prime}$ and $\beta$. For each realization we calculated the scattering strength $T(L=b)$ according to the algebraic expression.

3. After collecting a large number $(\approx 2000)$ of scattering strengths we calculated the corresponding histogram and normalized it. That yields an approximation for the distribution function $P_{L=b}(T)$ depending on the initial distribution $P_{0}(T)$. We also determined the average value $\langle T\rangle(L=b)$ and other quantities like median or geometric mean of the distribution.

4. We repeat the steps No. 2 and 3, but now drawing $T$ from the previously obtained distribution $P_{L=b}(T)$. We did this for each active scatterer independently. As a result we got a novel distribution which should coincide with $P_{L=b^{2}}(T)$ of the hierarchical $[b / V]$-model if the phases at each RG step can be taken as uncorrelated random phases. Indeed, the phases of $t(L=b), r(L=$ $b), t^{\prime}(L=b)$ turned out to be uniformly distributed in the interval [0,2 $\pi$ [ and correlations were not observed. Therefore, the 4 th step yields a reasonable approximation for $P_{L=b^{2}}(T)$ and, in particular, for $\langle T\rangle\left(L=b^{2}\right)$.

5 . Next, we repeat step No. $4 N$ times $(N \approx 20)$ which yields, for each initial distribution $P_{0}(T)$, the flow of the 
distribution function $P_{L}(T)$ and of the mean $\langle T\rangle(L)$ as functions of $\ln L=(N+2) \ln b$.

6 . We repeated the RG process for a number of different initial distributions $P_{0}(T)$.

An example for the flow of the distribution is displayed in Fig. 4 for a [3/4]-system with initial value $\langle T\rangle\left\langle T_{c} \approx\right.$ 0.6. It demonstrates that the distribution is attracted by the localization fixed point at $T=0$.

It generally turned out that after a few iterations the mean $\langle T\rangle$ was already a good scaling variable, i.e. once a certain mean value was reached, the flow of the distribution after further iterations did no longer depend significantly on the initial distribution. In particular we found for each model a certain distribution which was independent of $L$, characterized by $\langle T\rangle(L) \equiv T^{*}$ but unstable against changes $\langle T\rangle-T^{*} \neq 0$. Thus, we took the mean $\langle T\rangle$ to investigate the instability of the critical distribution. The critical exponent $\nu$ of the localization length is determined as

$$
\nu=\frac{\left(N-N_{0}\right) \ln b}{\ln \left\{\left(T(N)-T^{*}\right) /\left(T\left(N_{0}\right)-T^{*}\right)\right\}} .
$$

This expression follows from Eq. (1) with $X=\langle T\rangle$. The numbers $N_{0}$ and $N$ were chosen such that $\langle T\rangle$ was significantly repelled from $T^{*}$ without leaving the regime of power law repulsion.

In Figs. $5-8$ the elementary cells together with the critical distribution are shown. For the [3/5], [9/37]models also the flow of the average transmission is displayed. The critical distribution turned out to be broadly distributed over the interval $[0,1]$. Those models respecting the mirror symmetry of its elementary cell showed critical distributions approximately symmetric with respect to $0.5 \approx T^{*}$. The [3/4]-model breaks the mirror symmetry and $T^{*} \approx 0.6$, as previously found in [9]. This model and the [3/5]-model discussed in [8] are taken as a reference to judge the improvement in quantitative results when going to larger elementary cells. The symmetric critical distributions for different models are hardly distinguishable when taking numerical uncertainties into account. The critical distribution is not uniform over the interval $[0,1]$ (as conjectured in [18]), but develops a unique shallow minimum at 0.5 and has maximum values at the extreme positions $T=0,1$. This confirms the observations of [8] for the [3/5]-model and of [19] for the original CC model.

The values for the critical exponent $\nu$ are collected in Tab. 1. One observes that with increasing elementary cells the critical exponent $\nu$ approaches the CC network value in a monotonic way from above and the $[9 / 37]$ value, $\nu=2.75 \pm 0.3$, is already close to the value $\nu=2.43 \pm 0.18$ obtained for the original $\mathrm{CC}$ model [12]. Note that the value $\nu=3.5 \pm 0.3$ for the [3/5]model is compatible with the result $(\nu \approx 2.4)$ of 8 when taking the difference in scaling factors into account $(\ln 3 / \ln 2 \approx 1.58)$.

\section{MULTIFRACTAL ANALYSIS OF CRITICAL STATES}

A rather complete characterization of a LD transition phenomenon in terms of critical exponents is provided by the knowledge of the critical exponent $\nu$ of the localization length and the multifractal exponent $\alpha_{0}$ of critical states [2]. The exponent $\alpha_{0}$ describes how the typical value $P_{\mathrm{t}}$ of the probability $P$ to find an electron (being in that state) in a small box of linear size $l$ scales with respect to $\lambda=l / L$,

$$
P_{\mathrm{t}}(\lambda) \propto \lambda^{\alpha_{0}} .
$$

The fact that critical states have $\alpha_{0}>D$ in a $D$ dimensional system shows that critical states are extended but not totally 'space filling' (multifractal). Indeed, the typical value scales faster to zero as the average value $\left(\langle P\rangle \propto L^{-D}\right)$ and thus their ratio

$$
\hat{\rho}:=P_{\mathrm{t}} /\langle P\rangle \propto L^{-\left(\alpha_{0}-D\right)}
$$

is a convenient order-parameter for the LD transition. It vanishes algebraically on approaching the LD transition point from the extended phase and stays zero in the localized phase [2]. Also in the quantum Hall system, where no extended phase exists, $\alpha_{0}$ essentially determines the scaling of critical states, including energetic and spatial correlations.

The multifractal exponent $\alpha_{0}$ has been numerically determined in [13] for the original CC model and found to be $2.27 \pm 0.01$. The method used in that work can be described briefly as follows: The network model with its scattering matrices gives rise to a discrete unitary time evolution operator $U$ operating on bond amplitudes and mapping a state $\psi(t)$ at some time $t$ to a novel amplitude configuration $\psi(t+1)$ at one instant of time later by taking all of the scattering conditions into account. Energy eigenstates $\psi$ are solutions of

$$
U \psi=\psi
$$

and thus correspond to the eigenstates of $U$ with eigenvalue 1 . The energy of the underlying electron system enters here only parametrically through the scattering matrices and $U$ will have an eigenstate to eigenvalue 1 only for a discrete set of the parameter $T$. Instead of looking for this discrete set one can fix $T$ and ask for the more general eigenvalue problem

$$
U(T) \psi_{n}(T)=e^{i \phi_{n}(T)} \psi_{n}(T) .
$$

$\psi_{n}(T)$ are, for fixed $T$, a set of eigenstates at scattering strength $T$ of slightly modified disorder configurations, each of the modifications being an overall shift in random phases on the links.

The typical probability $P_{\mathrm{t}}(\lambda)$ can be identified as the geometric mean of local squared amplitudes integrated over boxes of size $l$, 


$$
P_{\mathrm{t}}(\lambda):=\exp \left\langle\ln \left(\sum_{\text {box }}|\psi|^{2}\right)\right\rangle .
$$

Here the average $\langle\ldots\rangle$ can be performed over different realizations and/or different local boxes within one realization.

We adopted this method for computing the critical exponent $\alpha_{0}$ of the hierarchical network models. The scattering strengths were fixed to the critical value $T^{*}$. We could not rely on a recursive calculation method, but needed some finite system of linear size $L>b$ where $b$ is the linear size of the elementary cell. This was necessary to detect the strong fluctuations of local amplitudes responsible for the multifractal behavior. Instead of going to very large sizes $L$ we took moderate sizes $(L \approx 50)$ but used several realizations to obtain $\alpha_{0}$ via Eqs. (11.7). An example for a critical wave function of a $[3 / 5]$-system is shown in Fig. 9. Strong spatial correlations, previously observed in critical states [20], are even more pronounced on the fractal support.

Since the hierarchical networks are defined on geometric fractals of dimension $D^{[b / V]}$ it is inappropriate to compare different values of $\alpha_{0}$ directly. Rather one should address the exponent of the order-parameter $\hat{\rho}$ which is $\alpha_{0}-D^{[b / V]}$. These values are listed in Tab. 1 . It can be seen that $\alpha_{0}-D^{[b / V]}$ monotonically approaches the CC model value from above as $D^{[b / V]}$ approaches $d=2$ from below. Since large values for $\alpha_{0}-D$ correspond to strong multifractal fluctuations of critical amplitudes this behavior tells that the hierarchical models with sparsely distributed active scatterers show more pronounced fluctuations than those with a denser set of active scatterers. For the [9/37]-model, however, we obtain $\alpha_{0}-D=0.33 \pm 0.05$ which is already close to the $\mathrm{CC}$ value, $0.27 \pm 0.01$.

\section{CONCLUSIONS}

We extended the concept of a real-space renormalization-group approach to the Chalker-Coddington model [8,9] in a systematic manner. We constructed hierarchical network models based on elementary cells which were cut out of a ChalkerCoddington model. Each elementary cell is characterized by the number $b^{2}$ of nodes and the number $V$ of active scatterers. The hierarchical models are fractal objects of dimension $D^{[b / V]}=\ln V / \ln b$ embedded in the original Chalker-Coddington model. In the $V=\propto b^{2} \rightarrow \infty$ limit the models coincide with the Chalker-Coddington model. We performed renormalization-group calculations for the following $[b / V]$-models: [3/4], [3/5], [5/13] and [9/37]. From these calculations we obtained the critical conductance distribution and the critical exponent $\nu$ of the localization length. The results for the [3/4] and [3/5]-models are consistent with those obtained in [8,9]. Our results for larger elementary cells show how fast the critical exponent approaches the value of the original ChalkerCoddington model.
Let us briefly comment on the possibility to use our method for a precise determination of the critical exponent of the original Chalker-Coddington model. For that purpose one not only has to use larger elementary cells, but also has to increase the number of realizations to get smoother histograms. Since the average value of $T$ turned out to be a good scaling variable the precision of the critical parameter for each hierarchical model can be improved by increasing the number of realizations in each iteration step. The critical parameters are then more precise for the particular hierarchical model, however have uncontrolled deviations from that of the ChalkerCoddington model. Better approximations can only be gained by increasing the size of the elementary cell and by studying the convergence of critical exponents. The computational effort then drastically increases; it is expected to be comparable to that of the well known finite size scaling method based on the transfer matrix technique [3].

To complete the characterization of the localizationdelocalization transitions in the hierarchical $[b / V]$ models we calculated the multifractal exponent $\alpha_{0}-$ $D^{[b / V]}$ of critical states and found that it approaches the Chalker-Coddington value from above when going to larger elementary cells. In summary, our work shows that a systematic renormalization-group treatment of networks of random quantum scatterers can not only reveal qualitative aspects of a possible localizationdelocalization transition, but also provides quantitative results for critical exponents the accuracy of which is controlled by convergence under stepwise increase of the size of elementary cells.

This work was supported by the Sonderforschungsbereich 341 of the Deutsche Forschungsgemeinschaft. We thank Boris Shapiro for stimulating us to this work and Daniel P. Arovas for helpful discussions. MJ thanks János Kertész for drawing his attention to compositions of elementary cells.

[1] P.W. Anderson, Phys. Rev. 109, 1492 (1958).

[2] M. Janssen, Phys. Reports 295 , 1 (1998).

[3] B. Kramer, A. MacKinnon, Rep. Prog. Phys. 56, 1469 (1993).

[4] Y.V. Fyodorov, A.D. Mirlin, Int. J. Mod. Phys. B 8, 3795 (1994).

[5] C.W.J. Beenakker, Rev. Mod. Phys. 69, 731 (1997).

[6] P.A. Lee, Phys. Rev. Lett. 42, 1492 (1979).

[7] H. Aoki, Solid State Commun. 31, 999 (1979).

[8] A.G. Galstyan, M.E. Raikh, Phys. Rev. B 56, 1422 (1997).

[9] D.P. Arovas, M. Janssen, B. Shapiro, Phys. Rev. B 56, 4751 (1997). 
[10] B. Shapiro, Phys. Rev. Lett. 48,823 (1982).

[11] J.T. Chalker, P.D. Coddington, J. Phys.C 212665 (1988).

[12] D.-H. Lee, Z. Wang, S. Kivelson, Phys. Rev. Lett. 70, 4130 (1993).

[13] R. Klesse, M. Metzler, Euro Phys. Lett. 32, 229 (1995).

[14] R. Prange, S. Girvin (Eds.), The Quantum Hall Effect, Springer, Berlin (1990).

[15] M. Janssen, O. Viehweger, U. Fastenrath and J. Hajdu, Introduction to the Theory of the Integer Quantum Hall Effect, VCH, Weinheim (1994).

[16] B. Huckestein, Europhysics Lett. 20, 451 (1992).

[17] Any smooth function $F(X)$ will serve equally well as a scaling variable $X^{\prime}=F(X)$ leaving the critical exponent $\nu$ universal.

[18] D.H. Cobdan, E. Kogan, Phys. Rev. B 54, 17316 (1996).

[19] Z. Wang, B. Jovanovic, D.-H. Lee, Phys. Rev. Lett. 77, 4426 (1996); S. Cho, P.A. Fisher, Phys. Rev. B 55, 1637 (1996).

[20] K. Pracz, M. Janssen, P. Freche, J. Phys.: Condensed Matter 8,7147 (1996).

\section{FIGURE CAPTIONS}

Figure 1: The graphical representation of the Chalker Coddington network model. Wave amplitudes propagating on links can be scattered either to the left or to the right by unitary scattering matrices situated at the nodes of the network.

Figure 2: Schematic representation of a ChalkerCoddington network of size $L \times L$ in a two-probe conductance measurement. The current flows from the source contact (down left) through the network to the drain contact (up right). The conductance is given as $G(L)=\left(e^{2} / h\right) T(L)$.

Figure 3: a) The construction of the [3/5]-hierarchical network. A $5 \times 5$ cell is cut out of the Chalker-Coddington model. Reducing the number of links to the outer world down to 4 links leads to an elementary cell with 5 active scatterers. The hierarchical construction yields a fractal network as a subset of the Chalker-Coddington model. At each level of the hierarchy the system size increases by a factor of 3. b) The renormalization scheme of Ref. [8]: 5 scatterers are replaced by one. In each step of the renormalization the rescaling factor is 2. c) The hierarchical model used in Ref. [9]. It can be viewed as a subset of the $[3 / 5]$-model with fully transmitting/reflecting central scatterers.

Figure 4: Flow of the distribution of transmission strengths under renormalization (in arbitrary units). The initial distribution (top) is peaked around a mean-value below the critical value $T^{*}$. After two renormalization steps (middle \& bottom) the distribution clearly approaches the stable distribution of full reflection $(T=0)$.

Figure 5: The elementary cell of the [3/4]-model (top) and the corresponding critical distribution of scattering strengths (bottom).

Figure 6: The elementary cell of the [3/5]-model (top left), the corresponding critical distribution of scattering strengths (top right) and the corresponding flow of average transmission strengths $T$ under increasing number $(N)$ of renormalization steps (bottom).

Figure 7: The elementary cell of the [5/13]-model (top) and the corresponding critical distribution of scattering strengths (bottom).

Figure 8: The elementary cell of the [9/37]-model (top left), the corresponding critical distribution of scattering strengths (top right) and the corresponding flow of average transmission strengths under increasing number $(N)$ of renormalization steps (bottom).

Figure 9: Squared amplitudes of a critical wave function for the [3/5]-model and a system size of $L=50$.

\section{TABLE CAPTIONS}

Table 1: Table of models $[b / V]$, fractal dimensions $D$, critical exponents of the localization length $\nu$ and of the order-parameter exponents $\alpha_{0}-D$. 


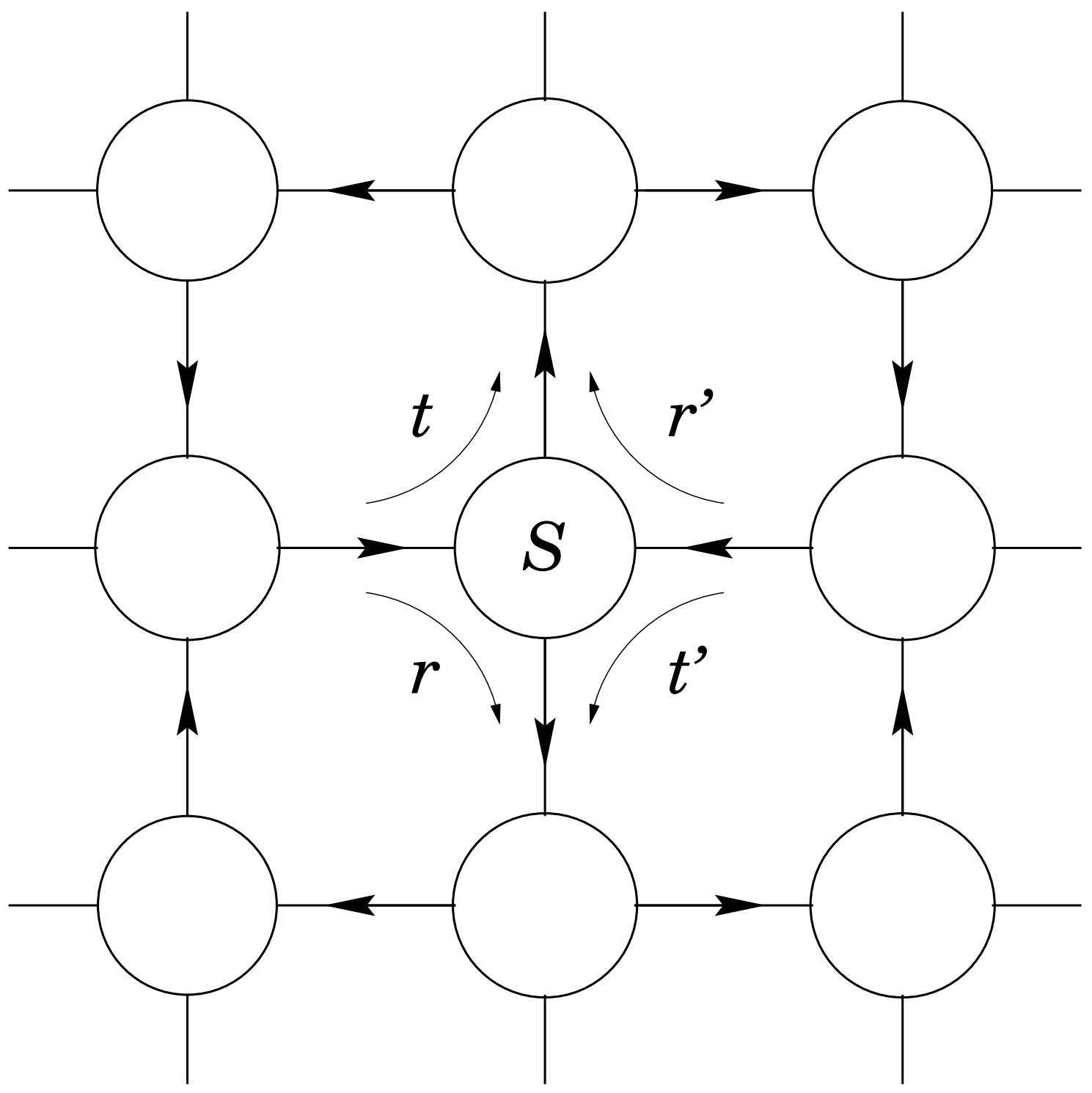




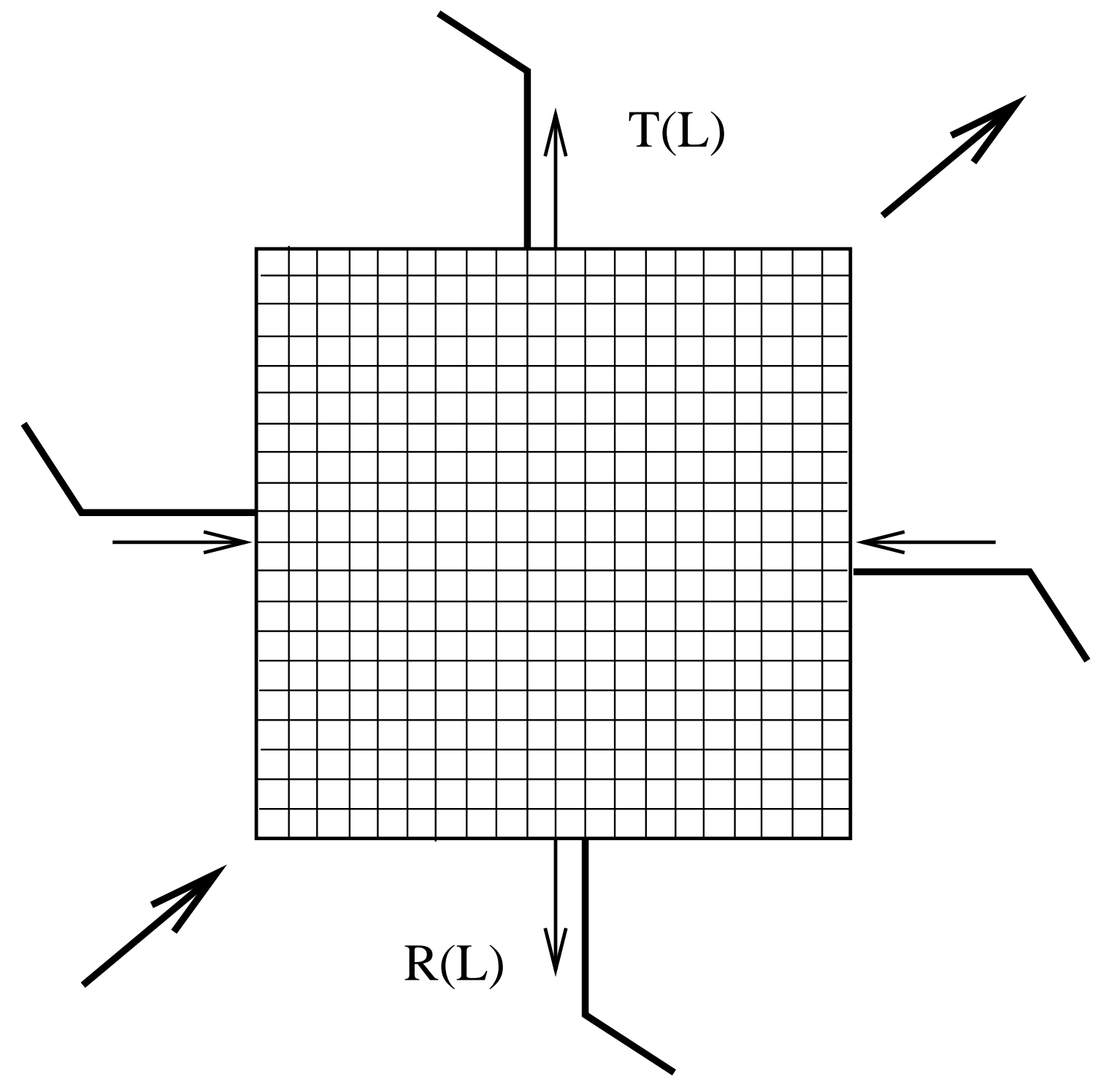



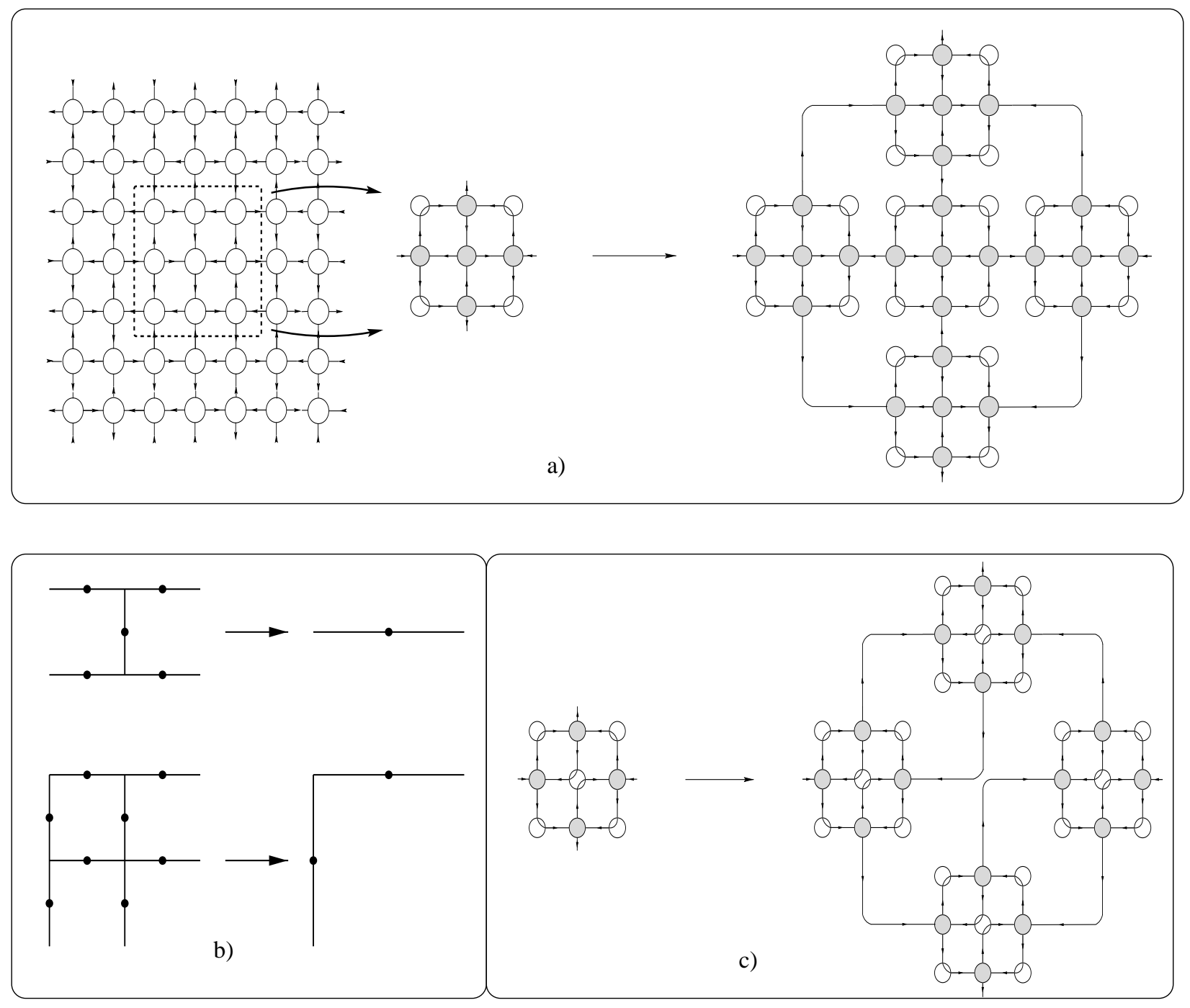

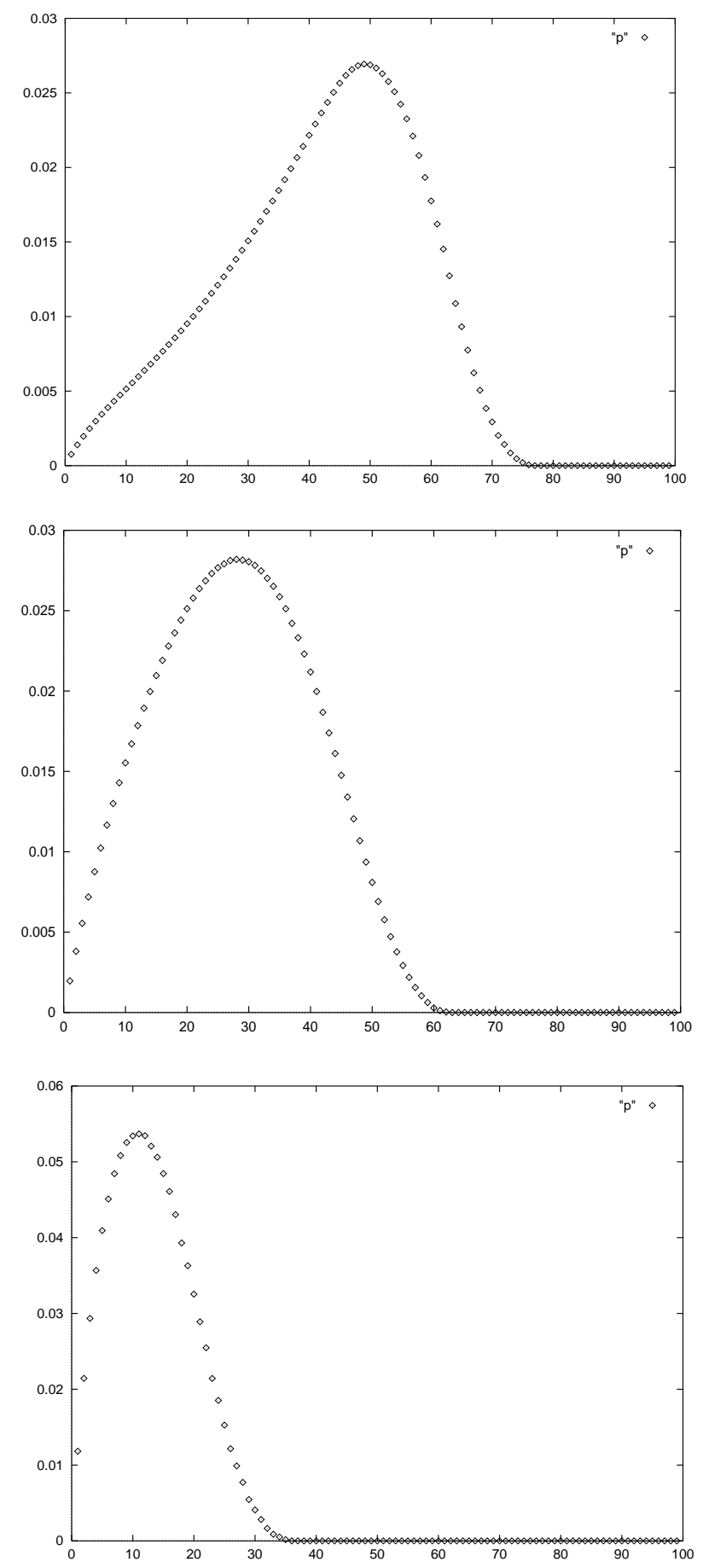

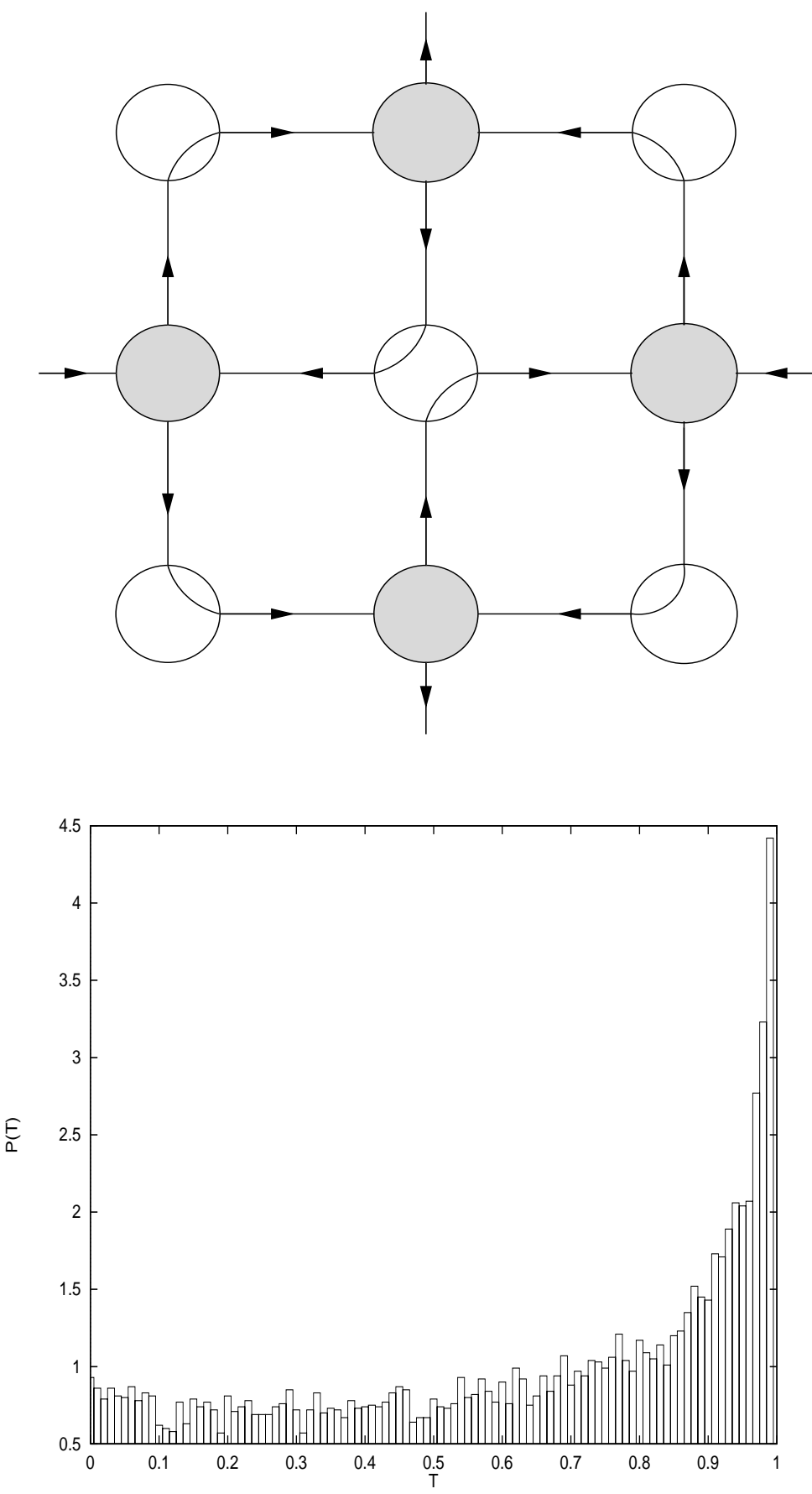

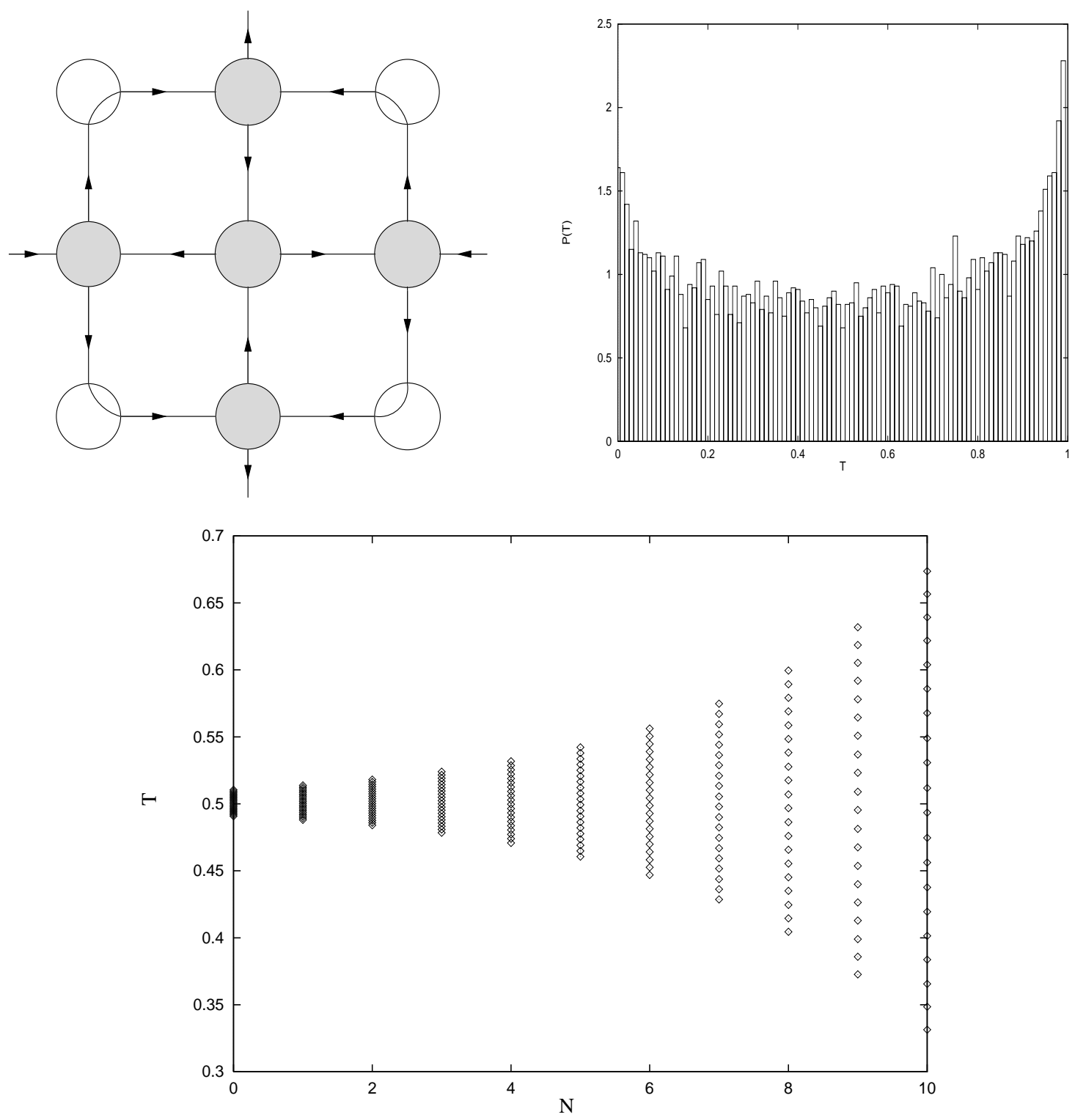

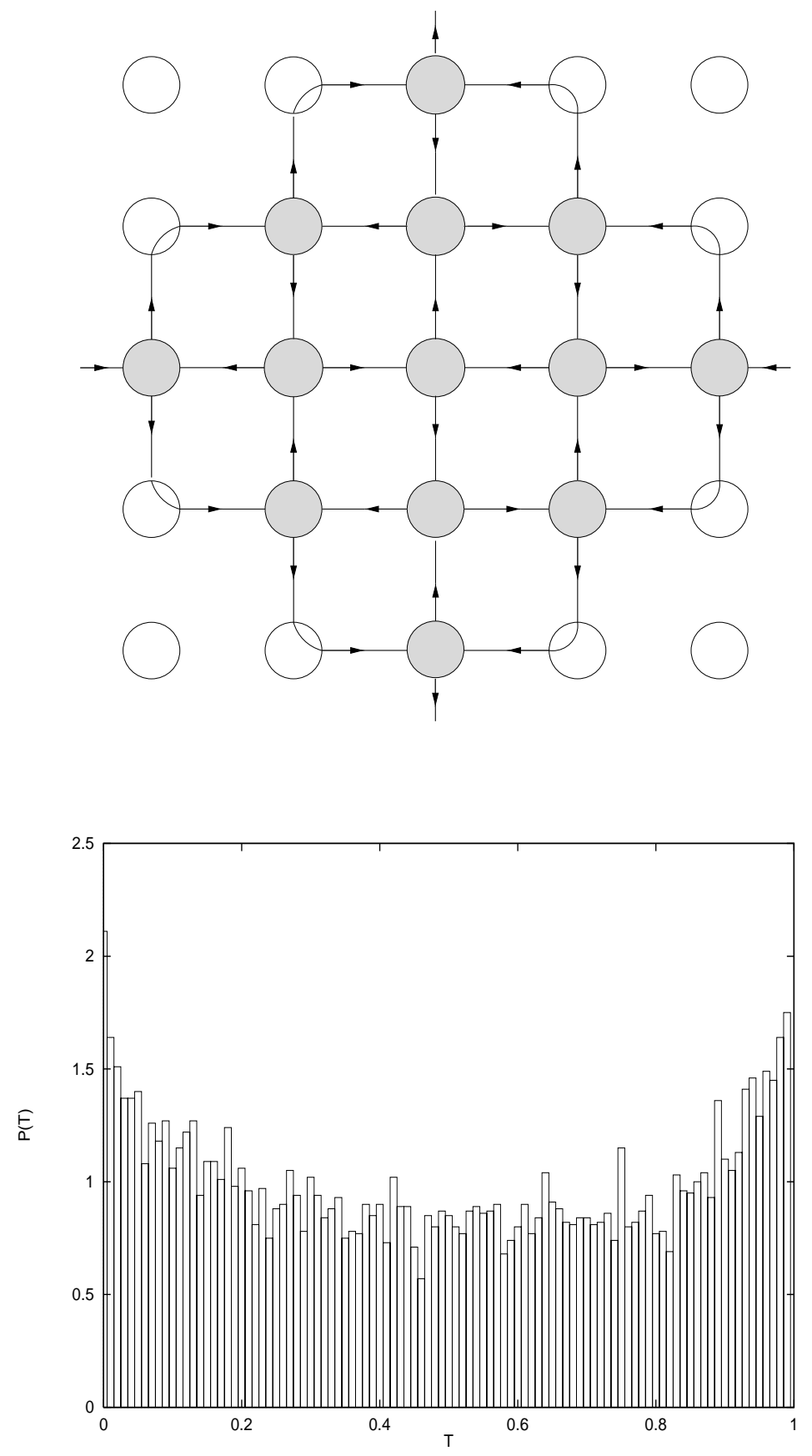

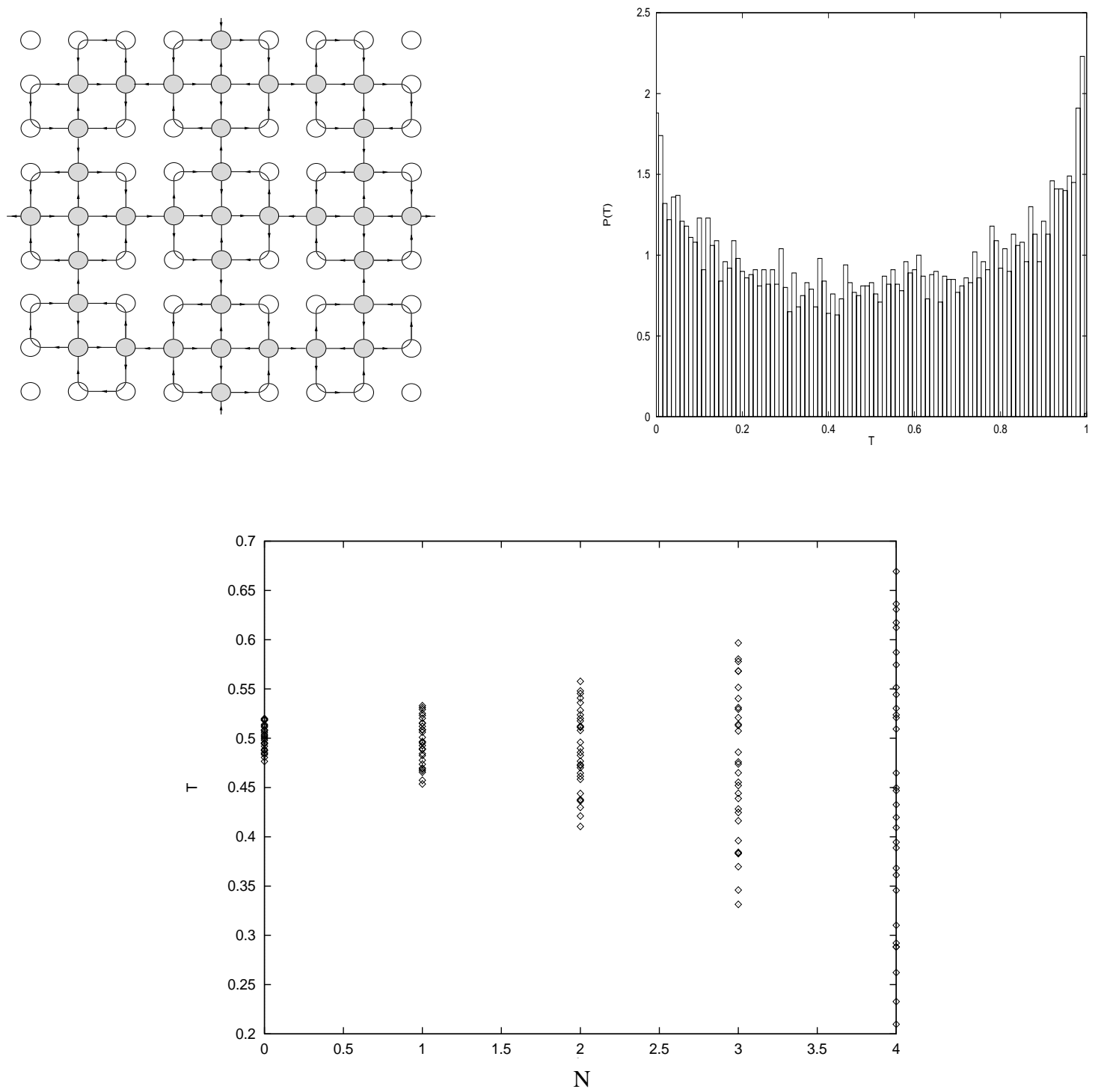


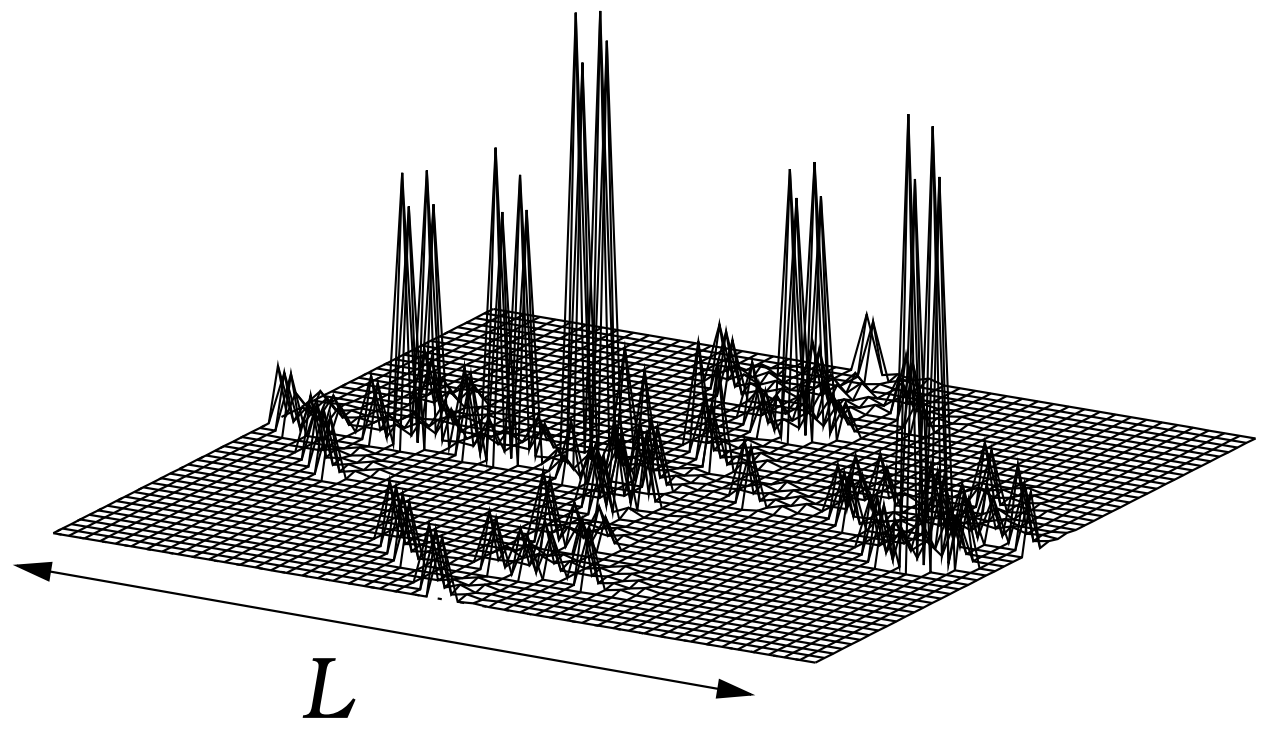




\begin{tabular}{|c|c|c|c|}
\hline Model $[b / V]$ & $D$ & $\nu$ & $\alpha_{0}-D$ \\
\hline \hline$[3 / 4]$ & $\approx 1.26$ & $6.6 \pm 0.6$ & $0.6 \pm 0.05$ \\
\hline$[3 / 5]$ & $\approx 1.46$ & $3.5 \pm 0.3$ & $0.4 \pm 0.05$ \\
\hline$[5 / 13]$ & $\approx 1.59$ & $2.9 \pm 0.3$ & $0.36 \pm 0.05$ \\
\hline$[9 / 37]$ & $\approx 1.64$ & $2.75 \pm 0.3$ & $0.33 \pm 0.05$ \\
\hline
\end{tabular}

Table 1: 